\title{
BAIKAL NEUTRINO PROJECT: HISTORY AND PROSPECTS
}

V. M. Aynutdinov, ${ }^{1}$ A. V. Avrorin, V. A. Balkanov, ${ }^{1}$

I. A. Belolaptikov, ${ }^{4}$ D. Yu. Bogorodskii, ${ }^{2}$ N. M. Budnev, ${ }^{2}$

R. Vishnevskii, ${ }^{5}$ I. A. Danil'chenko, ${ }^{1}$ G. V. Domogatskii, ${ }^{1}$

A. A. Doroshenko, ${ }^{1}$ A. P. D'yachok, ${ }^{2}$ Zh.-A. M. Dzhilkibaev, ${ }^{1}$

O. N. Gaponenko, ${ }^{1}$ K. V. Golubkov, ${ }^{4}$ O. A. Gress, ${ }^{2}$ T. I. Gress, ${ }^{2}$

O. I. Grishin, ${ }^{2}$ V. A. Zhukov, ${ }^{1}$ A. V. Zagorodnikov, ${ }^{2}$

A. M. Klabukov, ${ }^{1}$ A. I. Klimov, ${ }^{8}$ K. V. Konishchev, ${ }^{4}$

A. V. Korobchenko, ${ }^{2}$ A. P. Koshechkin, ${ }^{1}$ L. A. Kuzmichev, ${ }^{3}$

V. F. Kulepov, ${ }^{6}$ S. P. Mikheev, ${ }^{1}$ T. Mikolaiskii, ${ }^{5}$ E. Middell, ${ }^{5}$

M. B. Milenin, ${ }^{6}$ R. R. Mirgazov, ${ }^{2}$ E. A. Osipova, ${ }^{3}$ A. L. Pan'kov, ${ }^{2}$

L. V. Pan'kov, ${ }^{2}$ A. I. Panfilov, ${ }^{1}$ D. P. Petukhov, ${ }^{1}$ E. N. Pliskovskii, ${ }^{4}$

I. A. Portyanskaya, ${ }^{2}$ P. G. Pokhil, ${ }^{1}$ V. A. Poleshchuk, ${ }^{1}$ E. G. Popova, ${ }^{3}$

V. V. Prosin, ${ }^{3}$ M. I. Rozanov, ${ }^{7}$ V. Yu. Rubtsov, ${ }^{2}$ E. V. Ryabov, ${ }^{2}$

Yu. A. Semenei, ${ }^{2}$ S. I. Sinegovsky, ${ }^{2}$ B. A. Tarashchanskii, ${ }^{2}$

S. V. Fialkovskii, ${ }^{6}$ B. K. Shaibonov, ${ }^{4}$ A. V. Shirokov, ${ }^{3}$ and K. Shpiring ${ }^{5}$

The history, current status, and prospects for the development of the Baikal Neutrino Project are considered. The main physical results obtained with the help of NT200 and NT200+ neutrino telescopes are presented.

Keywords: neutrino telescope, high-energy atmospheric neutrinos, astrophysical neutrinos.

\section{INTRODUCTION}

By the present time, a huge volume of knowledge of the history of the development and structure of the Universe has been accumulated using optical, microvave, x-ray, and gamma-radiation telescopes. In the last few years, neutrino astronomy has become a new "window" into the Universe. Many astrophysical objects from typical stars like our Sun to active galactic centers are high-power sources of neutrinos with energies changing in a wide range. In the last decades, a number of underground facilities have been created (for example, see [1-4]) to investigate the energy spectrum of solar neutrinos (their characteristic energies are $0.2-15 \mathrm{MeV}$ ) and of other neutrino sources. As a result of these investigations, a model of thermonuclear fusion inside the Sun has experimentally been checked, a neutrino signal from a supernew explosion has been registered for the first time [3], and neutrino oscillations have been discovered that unambiguously indicate a nonzero neutrino mass [4]. The largest underground Super-Kamiokande facility [4] has a volume of $52000 \mathrm{~m}^{3}$, but even this volume is insufficient for registration of astrophysical neutrinos from many galactic and extragalactic sources, search for new forms of matter, and solution of other fundamental problems of astrophysics and elementary particle physics. The characteristic sizes of detectors solving these problems are about one cubic kilometer.

${ }^{1}$ Nuclear Research Institute of the Russian Academy of Sciences, Moscow, Russia; ${ }^{2}$ Irkutsk State University, Irkutsk, Russia; ${ }^{3}$ D. V. Skobel'tsyn Scientific-Research Nuclear Physics Institute at Moscow State University, Moscow, Russia; ${ }^{4}$ Joint Institute for Nuclear Research, Dubna, Russia; ${ }^{5}$ DESY-Zeuthen, Germany; ${ }^{6}$ Nizhnii Novgorod State Technical University, Nizhnii Novgorod, Russia; ${ }^{7}$ Saint Petersburg Marine State University, Saint Petersburg, Russia; ${ }^{8}$ Kurchatov Institute, Moscow, Russia, e-mail: nbudnev@api.isu.ru. Translated from Izvestiya Vysshikh Uchebnykh Zavedenii, Fizika, No. 6, pp. 52-61, June, 2010. Original article submitted March 1, 2010. 
Of principal importance for the development of high-energy neutrino astrophysics was M. A. Markov's suggestion to develop large optical detectors for high- and ultrahigh-energy neutrino registration in natural water reservoirs [5]. Natural ocean or deep sea water in these facilities serves simultaneously as a target for neutrinos, medium in which Cherenkov radiation of charged particles produced in the process of interaction is propagated, and protection from background cosmic rays. To register Cherenkov light, it is necessary to form a spatial array of highsensitive light detectors - optical modules - deeply under water. The possibility of practical implementation of this suggestion goes back to the middle 70s when the International Project on Muon and Neutrino Detector was launched near Hawaiian Islands (DUMAND). However, the practical implementation of a deep-water neutrino telescope in the open ocean appeared too complicated. As a result, after several unsuccessful attempts of arrangement of the DUMAND facility modules, Americans who played a key role in the Project decided that it is easier and more reliable to develop a facility for registration of high-energy neutrinos on the South Pole in the Antarctic ice. At first, the AMANDA facility was successfully developed [6]; it included 677 optical modules clamped to 19 cables in the ice bulk at depths of 800$2000 \mathrm{~m}$. At present, stage-by-stage arrangement of the IceCube facility with already 4800 optical modules clamped to 80 cables in a volume of about one cubic kilometer is being completed [7].

The development of the Baikal Neutrino Project was stimulated by A. E. Chudakov's suggestion to use the ice cover of the lake to mount a facility for deep-water neutrino registration. In the early 80 s it seemed that Lake Baikal is insufficiently deep for the arrangement of a large neutrino telescope primarily because of high atmospheric muon background. However, successes in the design and operation of the first Cherenkov detectors in Baikal, development of methods of detecting neutrino events against the muon background, and favorable environment provided the basis for the development of the first NT200 deep-water neutrino telescope [8] put into operation in 1998. In 2005, works on substantial increase of the effective volume and modernization of the Baikal Neutrino Telescope (NT200+ facility) were completed [9]. Nowadays, a start has been made of preliminary investigations aimed at the development of a gigaton underwater detector (NT1000) with a volume of about one cubic kilometer [10].

Natural water is an integral part of the Baikal Neutrino Telescope, and its properties (transparency, light background, temperature, etc.) and processes occurring in the lake (streams, sedimentation, etc.) influence significantly the facility operation; therefore, investigations of the facility environment from the very beginning occupied a significant place in the Baikal Neutrino Project. The development of new methods of continuous measurements at stationary buoy stations and of unique precision devices has allowed unique data on the hydrophysical and biogeochemical processes in Lake Baikal to be accumulated.

\section{BAIKAL DEEP-WATER NEUTRINO TELESCOPE}

The NT200 and NT200+ Baikal neutrino telescopes now in use (Fig. 1) are placed at a distance of $3.5 \mathrm{~km}$ from the coast in the $106^{\text {th }} \mathrm{km}$ of the Round-Baikal Railroad in the Southern part of Lake Baikal; the depth in this place is about $1370 \mathrm{~m}$. As a whole, the facilities represent a network of immersed buoy stations (BS) on which optical modules $(\mathrm{OM})$ registering Cherenkov radiation of relativistic charged particles produced due to neutrino interaction with matter, electronic modules intended for processing signals from the $\mathrm{OM}$ and communication with a coastal center, and various hydroacoustical, hydrooptical, hydrological, and other devices were placed. All BS have three basic structural elements: an anchor with a weight of 300-800 kg, a steel or synthetic rope fastened to it (in some cases, the so-called cable-rope), and one or several buoyancies - buoys, with the uppermost buoys at a depth of about $15 \mathrm{~m}$. In the first NT200 Baikal Deep-Water Neutrino Telescope (BDNT), 96 OM pairs are equally distributed between eight BS, forming the so-called garlands (Fig. 1); seven peripheral garlands of them are equidistantly distributed along a circle with a radius of $21.5 \mathrm{~m}$ and are clamped to the ends of glass fiber plastic pipes, which in their turn are clamped to a steel frame of the central garland of the facility at a depth of $1100 \mathrm{~m}$. The $\mathrm{OM}$ are glass spheres $50 \mathrm{~cm}$ in diameter with Kvazar photodetectors having a photocathode $37 \mathrm{~cm}$ in diameter placed inside of them [11]. The threshold of electronics triggering was adjusted to register single photons. To decrease the background load which can reach several hundred kilohertz and is caused by the Kvazar dark current and luminescence of the water environment, the OM are connected in pairs and operate in coincidence with a time window of $20 \mathrm{~ns}$. The OM pair forms a measuring channel. Two OM pairs with a glass sphere and electronics for primary OM signal processing, in particular, for generating 


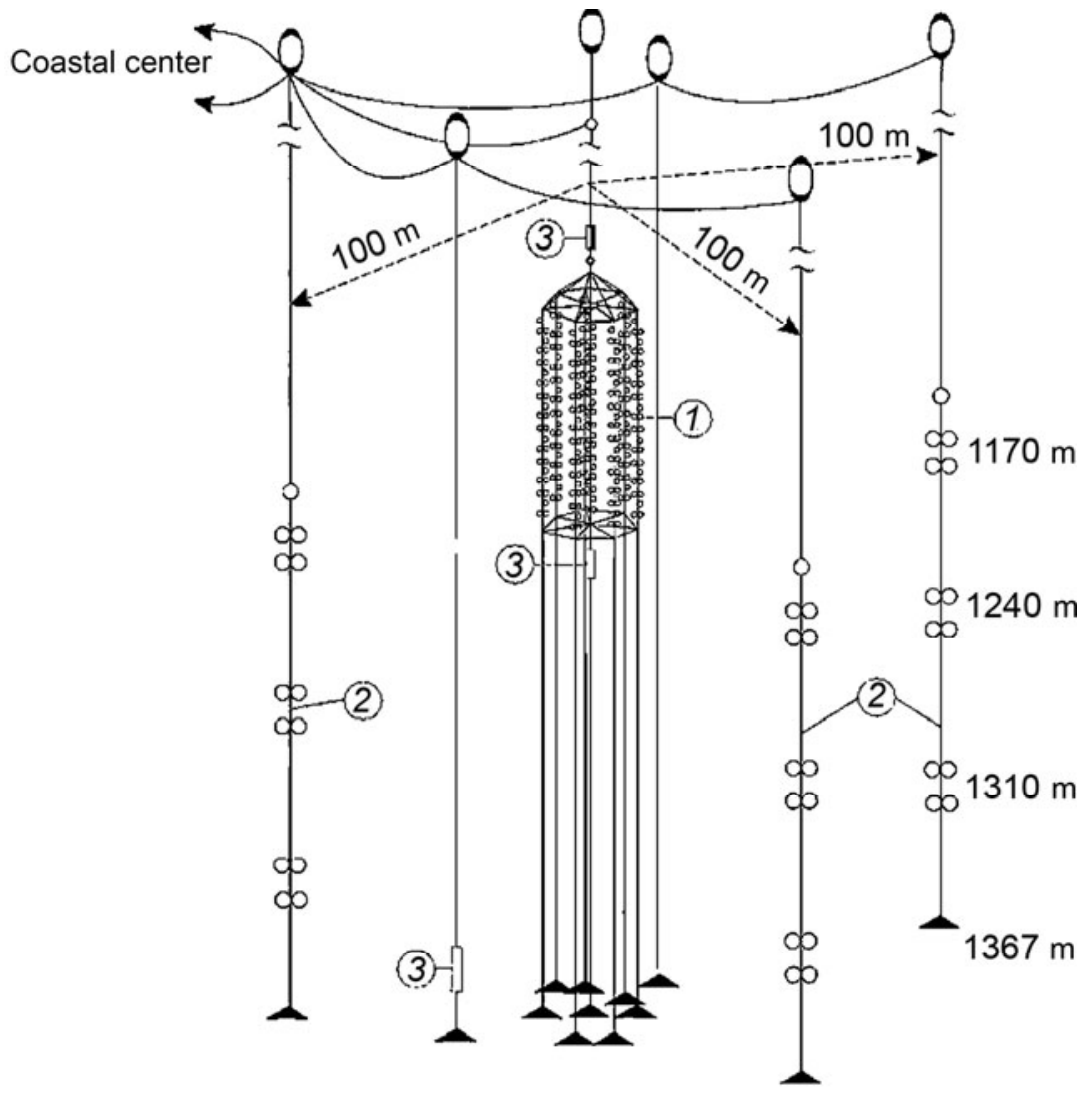

Fig. 1. Baikal Deep-Water Neutrino Telescope comprising NT200 Neutrino Telescope 1, external garlands of the NT200+ Neutrino Telescope 2, and alignment lasers 3.

signals about coincidence in the channels - local triggers (LT) - form a strap. All LT signals are fed to blocks of garland electronics (BGE) arranged on the same cable and serving three straps each. In this case, the leading LT signal front, the essence of the logic signal, bears information on time of channel triggering which is measured with an error of about several nanoseconds, and the LT duration is proportional to the total signal amplitude in the channel. Then LT signals enter a block of detector electronics (BDE) in which a control signal is generated when no less than three LT signals are registered during a time window of $500 \mathrm{~ns}$ (equal to about doubled time of muon passage through NT200). The BDE is mounted on the central garland together with several additional electronic modules providing information exchange with a coastal center (CC). The information on times and amplitudes of the triggered channels in the "dense" NT200 system allows various events to be reconstructed. For this purpose, spatial positions of all the OM of the facility must be known with an error no more than $20 \mathrm{~cm}$. These data are obtained with the help of a hydroacoustic system of measuring coordinates (HASMC) comprising six acoustic bottom beacons arranged along a circle with a radius of $600 \mathrm{~m}$ around NT200 together with devices that request beacons and receive answers [12].

The effective NT200 volume is insufficient for registration of ultrahigh-energy astrophysical neutrinos with sufficient statistics, which is the most important problem for all large-scale neutrino detectors in natural media. At the same time, a cascade of relativistic charged particles which generate $\sim 10^{12}$ photons is formed in the process of interaction of a high-energy (for example, $10 \mathrm{PeV}$ ) neutrino with water. In deep Baikal water, where the light scattering

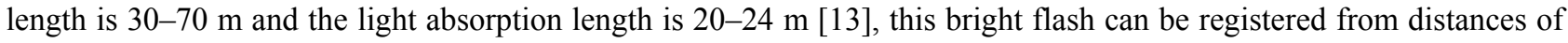
100-200 m. Therefore, a rare enough OM array with characteristic spacing of several tens of meters and even $100 \mathrm{~m}$ can be used to study ultrahigh-energy astrophysical neutrino fluxes in Baikal. Exactly this idea was used to develop the NT200+ Neutrino Telescope that includes, in addition to the NT200 Telescope, three more external garlands placed at vertices of an equilateral triangle at distances of $100 \mathrm{~m}$ from the NT200 center with 12 OM clamped to each garland. 
The same idea makes it possible to develop inexpensive external garlands of sensors in reasonable terms. In the process of developing NT200+, new ideology of information exchange between the coastal center and the underwater equipment of the Baikal Neutrino Telescope was experimentally verified. It is based on the application of commercial DSL-modems and the so-called one-board computers placed under water that has allowed the rate of information exchange through bottom cables to be increased by more than an order of magnitude; the same cables are used for power supply of the underwater equipment. One of the most important experimental problems is time synchronization of measuring channels of the underwater detectors with accuracy of a few nanoseconds. It is solved with the help of short light pulses from lasers illuminating the OM (Fig. 1).

\section{SOME RESULTS OBTAINED WITH THE BDNT}

The main problems solved within the framework of the Baikal Neutrino Project are the following:

- Investigation of characteristics of atmospheric muon fluxes.

- Identification of events from atmospheric neutrinos.

- Search for hypothetical particles (WIMP, heavy magnetic monopoles, etc.).

- Search for high-energy astrophysical neutrinos.

- Long-term monitoring of the hydrophysical processes in Lake Baikal.

To solve each problem, a special algorithm was adjusted which allowed events of the given type to be best identified in the total flow of information obtained with the help of the BDNT.

\subsection{Characteristics of atmospheric muon fluxes}

Muons generated in the process of meson and barion decay in the atmosphere due to the interaction of cosmic rays with air nuclei can pass several kilometers in water and form the main background for neutrino registration with the help of deep-water facilities. At a depth of $1 \mathrm{~km}$, the atmospheric muon flux exceeds the flux of muons generated by atmospheric muon neutrinos by about million times. On the other hand, the angular distribution and the curve of atmospheric muon absorption measured with the help of NT200 can be used to check models of the interaction of highenergy cosmic rays with nuclei, muon transmission through matter, and NT200 calibration. Figure 2 shows the atmospheric muon spectra expected at sea level calculated in [14] for the indicated high-energy nucleon-nucleus and meson-nucleus interaction models (QGSJET II, SIBYLL, etc.) and two variants of parameterization of the primary cosmic ray spectrum. Here the energy interval is shown where decays of charmed particles (curves 1-4) start to dominate as muon sources. Uncertainty of this contribution is imposed on the insufficiently investigated peculiarities of the cosmic ray spectrum near its "knee" with muon energies exceeding $3 \mathrm{PeV}$. Results of investigations of the muon fluxes are also important for calculation of the background for high-energy neutrino astronomy determined by atmospheric neutrinos. A detailed comparison of the muon fluxes calculated for the nuclear cascade model the same as for neutrinos (and with the same primary spectrum) with their measurements at sea level and under water will allow one to refine many unknown or poorly known parameters necessary for calculations of the nuclear cascade in the atmosphere and hence for a decrease of the degree of uncertainty in neutrino flux calculations.

\subsection{Identification of events from atmospheric neutrinos}

The main method of registering the atmospheric neutrinos (that is, neutrinos produced due to the interaction of cosmic rays with air nuclei) is a search for muons from the lower hemisphere that have passed through NT200. These muons are produced somewhere under the facility due to scattering on muon neutrino nuclei moving in the upward direction from below. Having processed the data for the period 1998-2002 (1008 observation days), we established 372 neutrino events [10]. This number must be compared to 385 events caused by the atmospheric neutrinos and background events from atmospheric muons expected according to the results of Monte Carlo simulation. 


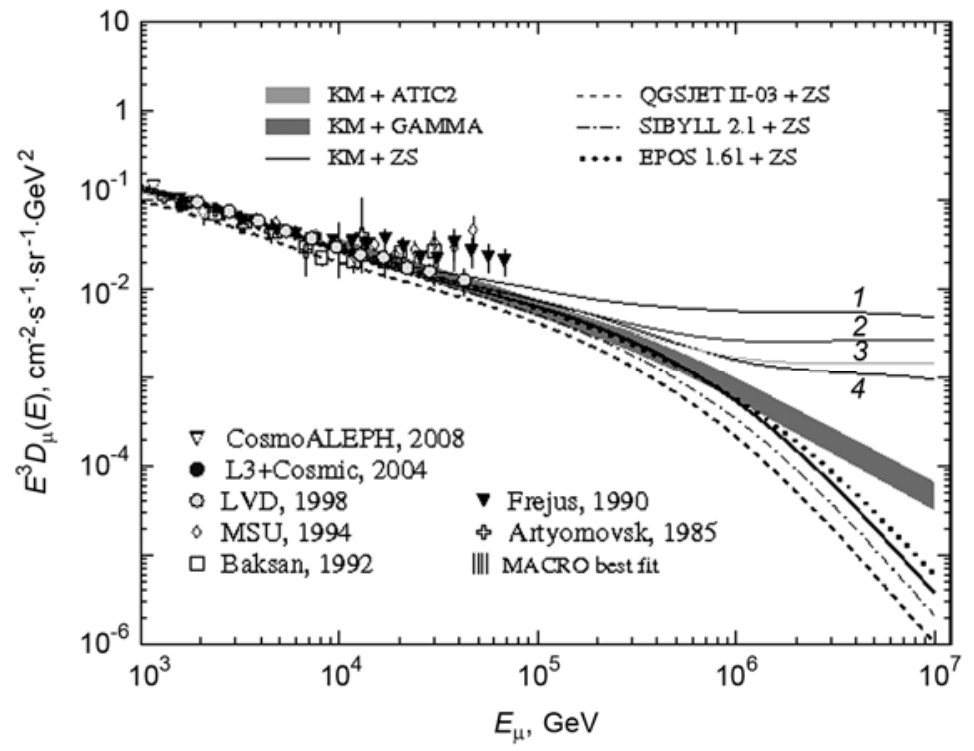

Fig. 2. Vertical spectrum of cosmic ray muons at sea level calculated for the indicated models of high-energy hadron interactions.

\subsection{Search for neutrinos from WIMP annihilation}

Weakly interacting massive elementary particles (WIMP) are the most popular candidates for the role of dark matter. The idea of their search with the help of the Baikal Neutrino Telescope is the following. If the WIMP do exist, they can be accumulated in the center of massive objects such as the Earth or the Sun and annihilate with a certain probability; moreover, the neutrinos must be among the end annihilation products. Within the framework of this hypothesis, a search for the neutrinos coming in the direction toward the Earth and the Sun centers must be conducted. To distinguish such events from the events registered by the Baikal Telescope from the Earth's center, special criteria were developed [15]. The energy threshold for this analysis was set $10 \mathrm{GeV}$, and the effective area was $1800 \mathrm{~m}^{2}$. During 1038 observation days, 48 muons with trajectories whose zenith angles $\theta$ were in the range $-1<\cos \theta<0.75$ had been registered. The expected number of events caused by the atmospheric muons in the case in which neutrino oscillations with the parameters $\delta M^{2}=2.5 \cdot 10^{-3} \mathrm{eV}$ and $\theta_{m} \approx \pi / 4$ is 56.6; without oscillations, it is 73.1 [10]. No muons coming from the Sun were detected [16].

\subsection{Search for fast magnetic monopoles}

Magnetic monopoles are hypothetical elementary particles of one more type. In the modern physical theory, the notion of the magnetic monopole was first introduced by Dirac in 1931. The charge of the Dirac monopole is $g=68.5 e$. With the velocity of motion in water close to the velocity of light in vacuum, the magnetic monopole emits the Cherenkov photons whose number is by a factor of 8300 greater than that of single muons (per unit length). The BGNT optical modules can register such bright objects from distances up to $100 \mathrm{~m}$; in this case, a large number of channels of the facility can be illuminated. On the other hand, monopoles with masses of $10^{7}-10^{14} \mathrm{GeV}$ remain relativistic even when they have passed the Earth. Therefore, the strategy of a search for the monopoles consists in identification of the events when the number of the triggered channels is no less than 30 and the object moves from the lower hemisphere. Then the selected events are checked with the help of special monopole criteria that allow us to 


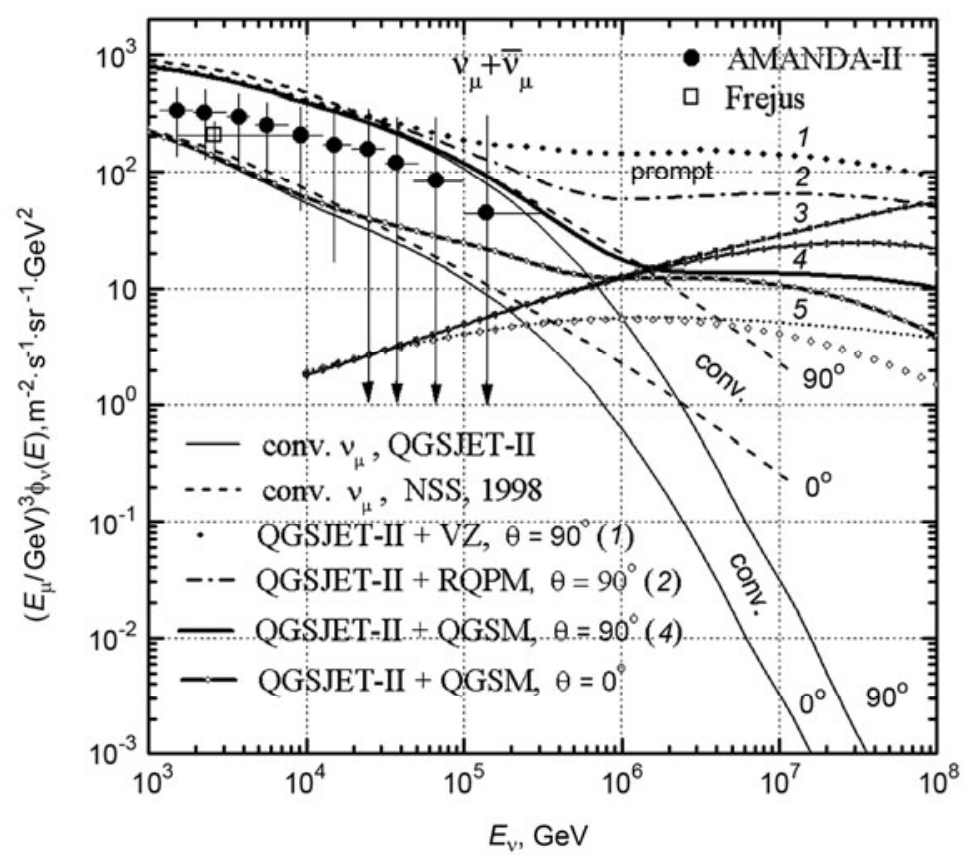

Fig. 3. Spectra of atmospheric high-energy muon neutrinos. Here symbols indicate measurements on Frejus and AMANDA-II facilities. Results of calculations for zenith angles $\theta=0^{\circ}$ and $90^{\circ}$, including neutrinos from the $\pi, K$ decay (conv.), are shown by the thin solid curves (the spectrum of primary cosmic rays for the Zatsepin-Sokol'skaya model) and results calculated in [18] with the Nikolskii spectrum are shown by the dashed curves. Here curves $1-5$ show the spectra of neutrinos from the decay of charmed particles (prompt) for the indicated models of charm production (see [14]).

exclude the background events caused by the passage of high-energy atmospheric muons, muon groups, and so on and, whenever possible, to identify the monopole events. During 1003 days of telescope operation, we did not reveal events that satisfied the magnetic monopole criteria. This have allowed us to establish the following restriction on the magnetic monopole flux: $F_{\text {mon }}<0.46 \cdot 10^{-16} \mathrm{~cm}^{-2} \cdot \mathrm{s}^{-1} \cdot \mathrm{sr}^{-1}$ for $v / c=1[17]$.

\subsection{Search for high-energy astrophysical neutrinos}

The central problem of neutrino telescopes in natural media is a search for ultrahigh-energy astrophysical neutrinos. Neutrinos generated by cosmic rays in the Earth's atmosphere form irreducible background for registration of neutrinos from remote astrophysical sources. Here it is important to note that the atmospheric neutrino flux (Fig. 3) decreases rather fast with increasing energy; therefore, for energies higher than $0.1-1 \mathrm{PeV}$, the possibility arises to seek and to detect neutrinos from astrophysical sources.

At high energies, difficulties of investigations of the background are due to the lack of the measured inclusive production cross sections of strange and charmed hadrons in nucleon-nucleus interactions for energies exceeding $10 \mathrm{TeV}$. For energies of about $100 \mathrm{TeV}$, it is expected that the main sources of atmospheric neutrinos and hence of the background for the astrophysical neutrinos will be decays of heavy short-living charmed mesons and barions. However, up till now no indisputable experimental confirmation of the existence of fast (prompt) atmospheric muons and neutrino has been achieved. The state of the art of the neutrino background problem is illustrated by Fig. 3. Here the results obtained on the AMANDA-II facility (the measurement errors for high energies are still very great) are shown together with the energy spectra of the muon neutrinos produced in the process of the $\pi, K$ (conv.) and $D, \Lambda_{c}$ (prompt) decays 


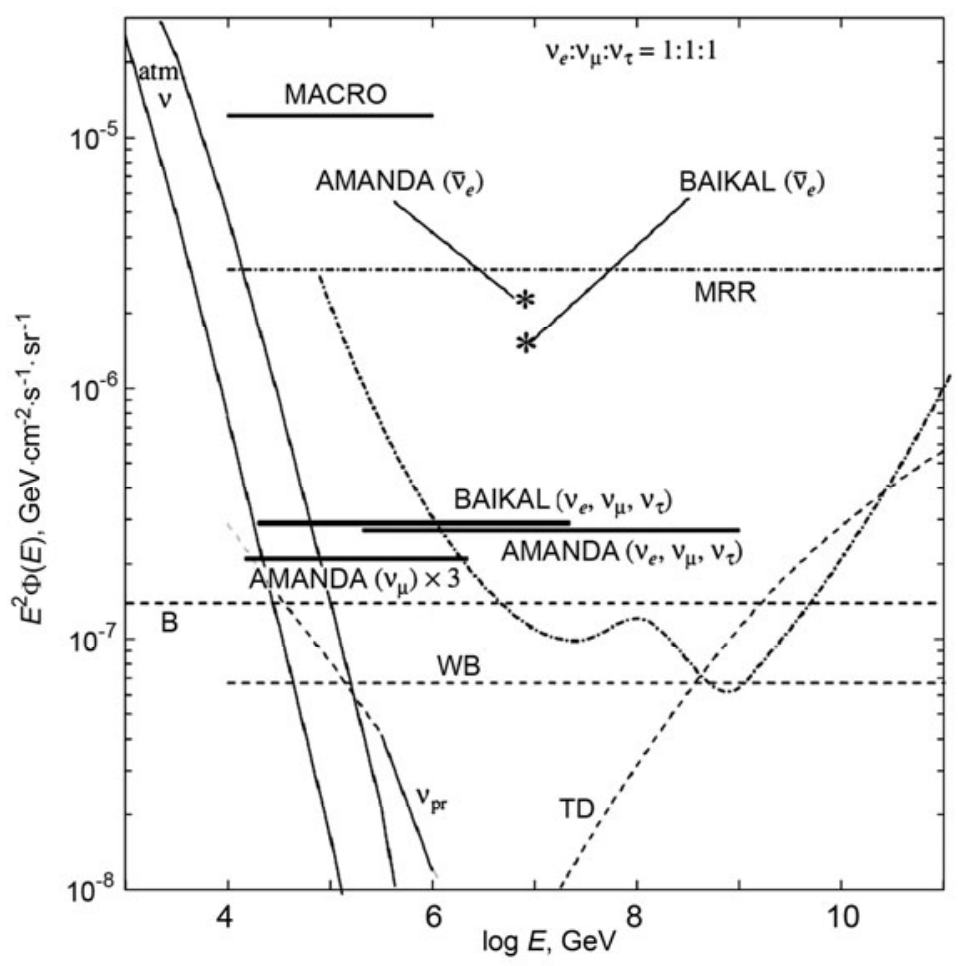

Fig. 4. Experimental and theoretical restrictions on the total diffusive flux of neutrinos of all types for a power-law spectrum $E_{v}^{-2}$.

calculated in $[14,18]$ for two zenith angles. The contribution of the last was calculated for the indicated models of charm production that predicted the neutrino fluxes whose magnitudes differed by 10-20 times.

The idea of the method is a search for signals from the cascade shallows arising due to neutrino interactions under the facility. The optical properties of the Baikal water are such that the effective volume required for registration and identification of cascades against the background at high energies significantly exceeds the volume of the facility.

For a power-law neutrino spectrum with the exponent equal to $2\left(\phi_{v} \sim E_{v}^{-2}\right)$ predicted by a number of models of cosmic ray acceleration for equal proportions of electron, muon, and tau neutrino fluxes, the limiting diffusive flux of astrophysical neutrinos of all types with energies $20 \mathrm{TeV}<E_{v}^{-2}<20 \mathrm{PeV}$ was obtained in [19]: $E_{v}^{2} \phi_{v}$ $<2.9 \cdot 10^{-7} \mathrm{~cm}^{-2} \cdot \mathrm{s}^{-1} \cdot \mathrm{sr}^{-1} \cdot \mathrm{PeV}$ (Fig. 4).

\section{PROSPECTS FOR THE BAIKAL NEUTRINO PROJECT}

\subsection{Gigaton water detector in Lake Baikal}

In the last few years, works and experiments are underway aimed at the development of the NT1000 Baikal facility [10] that will comprise 96 garlands 350-460 m long with 24 optical modules mounted on each garland and 2304 modules altogether. All garlands will be grouped into 12 clusters. The distance between the garlands will be about $60-$ $80 \mathrm{~m}$ (see Fig. 5). The effective NT1000 volume for registering cascades with energy $E>100 \mathrm{TeV}$ will be $\sim 0.5 \mathrm{~km}^{3}$, and the muon registration threshold will be 10-100 TeV. More exact facility parameters will be obtained by means of facility simulation using the Monte Carlo method. The new telescope will allow us to seek for ultrahigh-energy neutrinos on the level of the sensitivity to fluxes several times smaller than the Waxman-Bahcall (WB) limit, to 


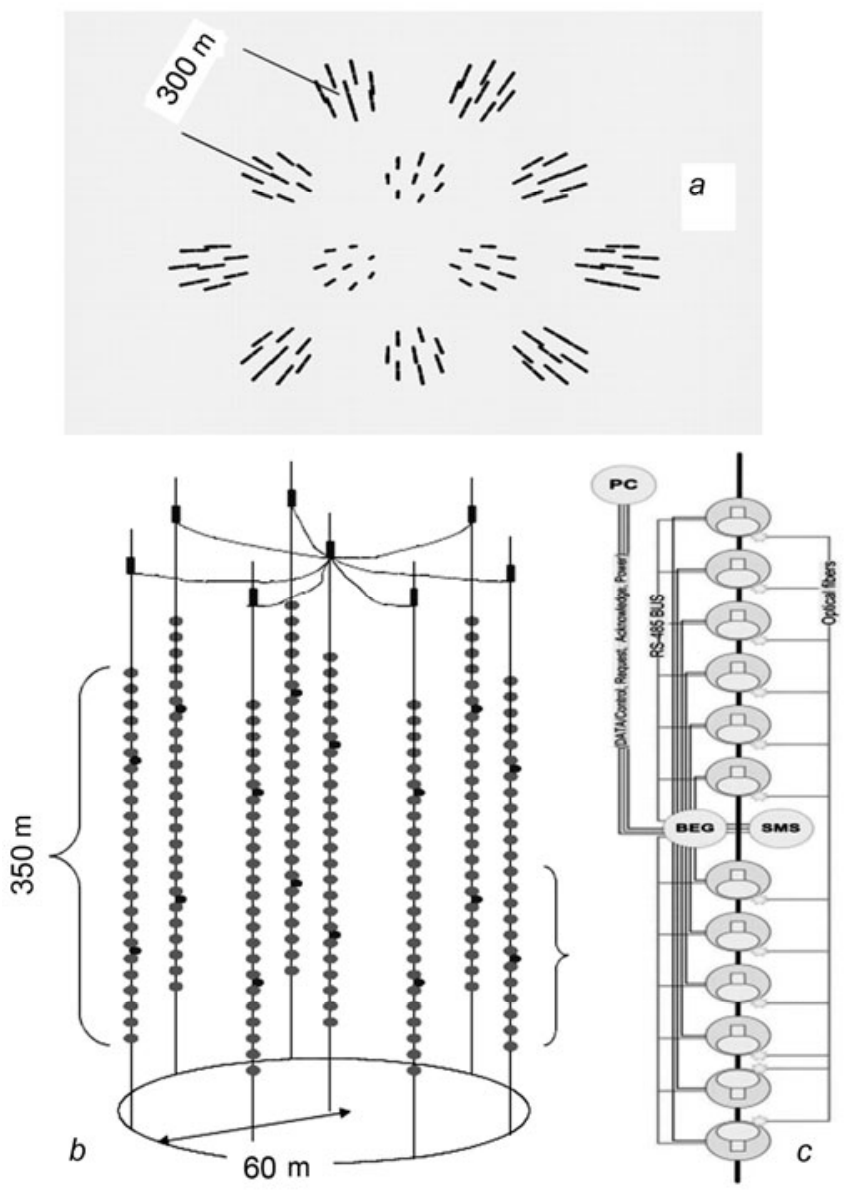

Fig. 5. Design of the gigaton water detector (GWD): $a$ ) top view with 12 clusters (2304 optical modules), $b$ ) cluster of the NT1000 facility (analogous to that of NT200), and $c$ ) experimental NT1000 garland.

decrease by more than an order of magnitude the limit for prompt magnetic monopoles or to register them, and to solve other problems of astrophysics and high-energy physics.

Photoelectron multipliers other than those used in NT200 will be used in NT1000, all electronic systems of the facility and methods of calibration, synchronization, and spatial positioning of optical modules will be organized differently. For practical implementation of new approaches, the first experimental garland of the NT1000 facility was built in 2008; it was essentially modernized in 2009. This garland includes 12 optical modules built around XP1807 (Photonis) and R8055 (Hamamatsu) photoelectron multipliers.

\subsection{Development of the acoustic registration method}

Detectors with sizes even greater than those of IceCube and NT1000 are necessary for registration of the highest-energy (exceeding $1000 \mathrm{PeV}$ ) neutrinos; at the same time, it is difficult to imagine an optical detector whose volume is much greater than one cubic kilometer. In [20] it has been demonstrated that bipolar acoustic pulses with duration of a few tens of microseconds are generated by high-energy showers in water. This effect can be used to register high-energy neutrinos in sea or Lake Baikal water. The expected amplitude of pulses generated in Baikal water by the shower having energy of $10^{19} \mathrm{eV}$ is about $10 \mathrm{mPa}$ at a distance of $100 \mathrm{~m}$ in the direction perpendicular to the 


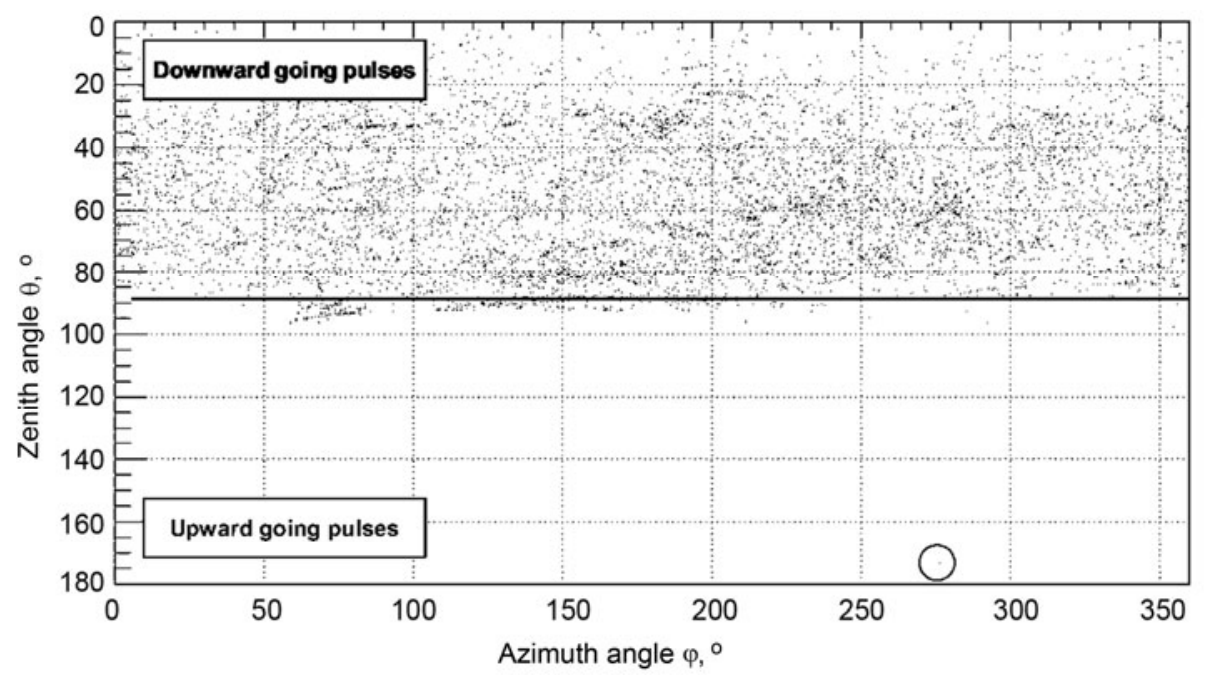

Fig. 6. Distribution of the registered bipolar pulses over the zenith angles.

shower axis. Among the advantages of the method is weak sound attenuation in water; therefore, the acoustic detector of ultrahigh energy neutrino can in principle have much greater effective volume for the number of measuring channels the same as in the Cherenkov facility.

Investigations aimed at elucidation of the possibilities of acoustic registration of ultrahigh-energy neutrinos in Lake Baikal go back to 2000. Since then large experience in measuring acoustic signals and reconstructing coordinates of their sources has been accumulated, and the main features of the background have been investigated [21]. With allowance for the accumulated knowledge, a 4-channel deep-water digital device has been developed and manufactured [22]. It can be considered as a prototype of the module of the future deep-water acoustic neutrino detector. The antenna of the device comprises four hydrophones placed in vertices of a regular tetrahedral pyramid with edge length of $1.5 \mathrm{~m}$. Amplified hydrophone signals are digitized with a time step of $5 \mu$ s and analyzed under water with the help of a NovaC400 microcomputer. Rare neutrino-like pulses of small duration $(\tau<50 \mu \mathrm{s})$ are continuously monitored against the constant noise background of the medium with the help of a special algorithm. It allows the direction on quasi-local sound pulse sources to be reconstructed with accuracy of about $1^{\circ}$ and the background caused by signal imitation by other sources to be suppressed significantly.

In 2006, the device was first mounted stationary at a depth of $100 \mathrm{~m}$. The small depth is chosen "to hear" deep lake layers where the number of sound sources is small, at least in Baikal. To reduce the acoustic background from the upper hemisphere, sound-proof caps were mounted above the hydrophones. Result of preliminary processing of experimental data demonstrate that up to now, we have detected only one bipolar pulse arrived from below (Fig. 6) against the significant background produced by noise pulses from the upper hemisphere (noise from waves, navigation, ice shift and cracking, etc.) and arrived at small angles to the horizon from the lower hemisphere (caused by refraction).

\section{INVESTIGATION OF THE DYNAMIC PROCESSES IN LAKE BAIKAL}

Within the framework of the Baikal Neutrino Project, radically new possibilities for investigations of the dynamic processes in the lake have appeared based on technologies of arrangement from ice of submerged buoy stations, including stations connected with the coastal center by communication cable, fabrication of high-sensitivity devices, and application of new observation methods. As a result of our investigations, the phenomenon of Baikal water luminescence was discovered, its nature was investigated, the method of luminescence application for a study of the hydrophysical processes was suggested, and the optical Baikal water properties were investigated in detail [23]. In cooperation with EAWAG (Switzerland) and DESY-Zeuthen (Germany), new data on the horizontal and vertical exchange of Lake Baikal waters were obtained with the help of hydrophysical devices distributed over three buoy 
stations, for example, it was experimentally proved that the main contribution to deep water refreshment comes from near-slope water descent initiated by the atmospheric processes [24].

This work was supported in part by the Russian Foundation for Basic Research (grants Nos. 08-02-00432-a, 09-02-10012-k, 07-02-00791, 07-02-00791, 08-02-00198, 09-02-10001-k, 09-02-00623-a, and 09-02-12295), Federal Agency for Science and Innovations (State Contract No. 02.740.11.0018), Federal Agency of Education (State Contract No. 1242 and Projects RNP.2.2.1.1.7334, 2.2.1.1/1483, 2.1.1/1539, and 2.2.1.1/5901), Baikal Scientific-Educational Center, and by the President of the Russian Federation under Program "Leading Scientific Schools of the Russian Federation" (grants NSh-321.2008-2 and NSh-1027.2008-2).

\section{REFERENCES}

1. $\quad$ B. T. Cleveland et al., ApJ., 496, 505-529 (1998).

2. M. Koshiba, Phys. Rep., 200, Nos. 5-6, 229-381 (1992).

3. R. D. McKeown and P. Vogel, Phys. Rep., 394, 315-356 (2004).

4. T. Kajita, Prog. Phys., 69, 1607-1635 (2006).

5. M. A. Markov, in: Proc. 1960 Annual Int. Conf. on High Energy Phys., Rochester (1960), p. 578.

6. F. Halzen, New Astron. Rev., 42, 289-299 (1998).

7. J. Ahrens et al., Astropart. Phys., 20, 507 (2004); T. De Young, Mod. Phys. Lett., A24, 1543-1557 (2009); arXiv:0906.4530.

8. I. A. Belolaptikov et al., Astropart. Phys., 7, 263 (1997).

9. V. M. Aynutdinov et al., Nucl. Instrum. Methods, A567, 433-437 (2006).

10. V. M. Aynutdinov et al., Nucl. Instrum. Methods, A588, 99-106 (2008).

11. R. I. Bagduev et al., Nucl. Instrum. Methods, A420, 138-154 (1999).

12. V. M. Aynutdinov et al., Akust. Zh., 55, No. 6, 721-731 (2005).

13. V. Balkanov et al., Nucl. Instrum. Methods, A298, 231-239 (2003).

14. A. A. Kochanov, T. S. Sinegovskaya, and S. I. Sinegovsky, in: Proc. $31^{\text {st }}$ ICRC, Lodz (2009). OG 2.50693; HE 2.10962; arXiv:0906.3791; arXiv:0906.0671.

15. V. Balkanov et al., Nucl. Phys. (Proc. Sup.), B91, 438 (2001).

16. A. Avrorin et al., in: Proc. $31^{\text {st }}$ ICRC, Lodz (2009) (icrc1165); arXiv:0909.5589.

17. K. Antipin et al., Astropart. Phys., 29, 366-372 (2008).

18. V. A. Naumov, T. S. Sinegovskaya, and S. I. Sinegovsky, Nuovo Cimento, A111, 129-148 (1998).

19. V. Aynutdinov et al., Astropart. Phys., 25, 140-150 (2006).

20. G. A. Askariyan and B. A. Dolgoshein, Pis'ma Zh. Eksp. Teor. Fiz., 25, No. 5, 232-233 (1977); G. A. Askariyan et al., Nucl. Instrum. Methods, 164, No. 2, 267-278 (1979).

21. V. M. Aynutdinov et al., Akust. Zh., 52, No. 5 (2006).

22. V. M. Aynutdinov et al., Int. J. Mod. Phys., A21, Suppl. 01, 202-206 (2006).

23. L. B. Bezrukov et al., Izv. Ross. Akad. Nauk, Ser. Fiz. Atm. Okeana, 34, No. 1, 97-103 (1998).

24. M. Schmid, N. M. Budnev, N. G. Granin, et al., Geophys. Res. Lett., 35, L09605 (2008), doi:10.1029/2008GL033223. 